\title{
Identification and characterization of heat-responsive microRNAs at the booting stage in two rice varieties 9311 and Nagina 22
}

\begin{tabular}{|c|c|}
\hline Journal: & Genome \\
\hline Manuscript ID & gen-2020-0175.R1 \\
\hline Manuscript Type: & Article \\
\hline $\begin{array}{r}\text { Date Submitted by the } \\
\text { Author: }\end{array}$ & 14-Apr-2021 \\
\hline Complete List of Authors: & $\begin{array}{l}\text { Luo, Ying; Hunan Agricultural University, College of biological science } \\
\text { and technology } \\
\text { Wang, Tao; Hunan Agricultural University } \\
\text { Yang, Dan; Hunan Agricultural University } \\
\text { Luo, Biao; Hunan Agricultural University } \\
\text { Wang, Weiping; State Key Laboratory of Hybrid Rice, Hunan Hybrid Rice } \\
\text { Research Center } \\
\text { Yu, Dong; State Key Laboratory of Hybrid Rice, Hunan Hybrid Rice } \\
\text { Research Center } \\
\text { He, Fulin; Hunan University of Science and Engineering } \\
\text { Wang, Qiming; Hunan Agricultural University } \\
\text { Rao, Liqun; Hunan Agricultural University }\end{array}$ \\
\hline Keyword: & $\begin{array}{l}\text { rice, heat-responsive, microRNA, booting stage, high-throughput } \\
\text { sequencing }\end{array}$ \\
\hline $\begin{array}{r}\text { Is the invited manuscript for } \\
\text { consideration in a Special } \\
\text { Issue? : }\end{array}$ & Not applicable (regular submission) \\
\hline
\end{tabular}

\section{SCHOLARONE ${ }^{\text {tw }}$ Manuscripts}




\section{Identification and characterization of}

2 heat-responsive microRNAs at the booting

3 stage in two rice varieties 9311 and Nagina

$4 \quad 22$

5 Ying Luo, Tao Wang, Dan Yang, Biao Luo, Wei-Ping Wang, Dong Yu, Fu-Lin He,

6 Qi-Ming Wang, and Li-Qun Rao

7

8

Y. Luo*, T. Wang*, D. Yang, B. Luo, Q.-M. Wang, and L.-Q. Rao. College of Bioscience and Biotechnology, Hunan Agricultural University, Changsha 410125, China.

W.-P. Wang, and D. Yu. State Key Laboratory of Hybrid Rice, Hunan Hybrid Rice Research Center, Changsha 410125, China.

Y. Luo*, and H.-L. He. College of Chemistry and Bioengineering, Hunan University of Science and Engineering, Yongzhou 425199, China.

Corresponding authors: L.-Q. Rao (email: liqunrao@163.com); Q.-M. Wang (email: wqmqmx21

@126.com)

${ }^{*}$ These authors contributed equally to this work. 
Abstract: MicroRNAs (miRNAs) are small, non-coding, regulatory RNAs that play important roles in abiotic stress responses in plants. but their regulatory roles in the adaptive response to heat stress at the booting stage in two rice varieties 9311 and Nagina 22, remain largely unknown. In this study, 464 known miRNAs and 123 potential novel miRNAs were identified. Of these miRNAs, a total of 90 differential expressed miRNAs were obtained with 9311 libraries as control group, of which 54 upregulated and 36 downregulated miRNAs. To gain insight into functional significance, 2773 potential target genes of these 90 differentially expressed miRNAs were predicted. GO enrichment showed that the predicted target genes of differentially expressed miRNAs including NACs, LACs, CSD, and Hsp40. KEGG pathway analysis showed that target genes of these differentially expressed miRNAs were significantly enriched in plant hormone signal transduction pathway. The expression levels of ten differentially expressed miRNAs and their target genes obtained by qRT-PCR were largely consistent with the sequencing results. This study lays a foundation for the elucidation of the miRNA-mediated regulatory mechanism in rice at elevated temperatures.

Key words: rice, heat-responsive, microRNA, target gene, booting stage, high-throughput sequencing

\section{Introduction}

Climate change is one of the severe challenges for global agriculture in the 21 st century. According to the (Intergovernmental Panel on Climate Change) IPCC (2013), the globally averaged combined land and ocean surface temperature rose by $0.85{ }^{\circ} \mathrm{C}$ over the period 1880 to 2012, and is expected to continue to rise by $0.3{ }^{\circ} \mathrm{C}$, which could reach $1.0-3.7^{\circ} \mathrm{C}$ by the end of the 
century. With the warming of the climate, high temperature occurs frequently all over the world, and the distribution range is also gradually expanding. Heat stress can be defined as a rise in temperature beyond a threshold level for a period of time, sufficient to cause irreversible damage to plant growth and development (Wahid and Close 2007). Sudden rises in temperature to high levels may lead to cell death within a few minutes as a consequence of extensive protein denaturation and aggregation and loss of membrane integrity (Wahid and Close 2007). Furthermore, prolonged exposure to moderately high temperatures can lead to reduced cellular function and overall plant fitness (Bokszczanin and Fragkostefanakis 2013). It had showed that maize production were significantly reduced when the maximum temperature on the day exceeds $32{ }^{\circ} \mathrm{C}$ (Hawkins et al. 2013). When temperatures exceed the current temperature of more than 2 ${ }^{\circ} \mathrm{C}$, global crop yields will be significantly reduced, especially at low latitudes, with yields reduced by more than $20 \%$ (Lobell and Burke 2010). In the summer of 2003, global climate continues to warm on a steady scale, heat stress happened in Eurasian Continent, and the yields of wheat and maize decreased significantly (Ciais et al. 2005). In 2012, a long period of heat and drought stress in the central part of the United States led to a serious loss of corn yield (LeComte 2013), and rice is often damaged by heat stress at flowering stage and early grain filling stage in Asia (Wassmann et al. 2009). However, plants can be able to perceive and respond to temperature changes, and a series of heat-responsive mechanisms have been formed in the course of long-term evolution. so it is important to explore how rice responds to heat stress.

MiRNAs in plants, single-stranded non-coding RNA molecules with a length of 19-24 nucleotides, serve as gene expression regulators of target messenger RNAs (target mRNAs) at the post-transcriptional levels (Dugas and Bartel 2004). MiRNA coding genes are transcribed by RNA 
polymerase II (Pol II) to generate primary miRNAs (pri-miRNAs) that fold into characteristic self-complementary stem-loop secondary structures (Xie et al. 2005). The pri-miRNAs are cleaved at least twice by Dicer-like1 (DCL1) endonucleases to yield the precursor-miRNAs (pre-miRNAs) that are further processed to release miRNA/miRNA* duplexes. Mature miRNAs are loaded onto Argonaute 1 (AGO1) to form miRNA-induced silencing complexes (miRISCs) that guide target-specific mRNA cleavage or translational repression (Cui et al. 2017). There are many regulation mechanisms of plant miRNA, the study found that a significant increase in target mRNA content in agol mutants with over-expression of the AGO1 gene in Arabidopsis thaliana, and this indicated that the cleavage of the miRNA target gene was regulated by the $A G O 1$ gene (Achard et al. 2004). For example, miR159 in Arabidopsis thaliana regulates the synthesis of LEAF protein by cleaving GAMYB mRNA, which affects the formation and development of flower organs, resulting in the delay of flowering period (Achard et al. 2004). Small number of plant miRNAs are not fully paired with their transcription products of target mRNA, but miRNA can regulates gene expression by inhibiting target mRNA translation (Li et al. 2013). MiRNA can also mediate the production of ta-siRNA (trans-acting siRNA) (Allen et al. 2005). Furthermore, a 24nt long miRNA (ImiRNA) was found in rice, which was cut by DCL3 and interacted with AGO4 subfamily proteins. lmiRNA did not regulate the target gene at the post transcriptional level, but inhibits its expression at the transcriptional level by mediating DNA methylation of the target gene (Wu et al. 2010). Thus, it revealed a new regulation mode of miRNA on target genes.

Rice is the most important food crop in the world, and environmental factors directly affect its yield and quality. MiRNAs in plant regulate related genes after transcription under temperature stress, and the target genes of many miRNAs are transcription factors (TFs). For example, miR160, 
miR164, and miR397 families can response to cold stress and regulate physiological processes through ARF, NAC, ICE1 and other TFs (Liu et al. 2007; Wang et al. 2005). MiR160 could effectively affect seed germination, seedling development and root cap formation by negatively regulating the expression of $A R F$ (Liu et al. 2007; Wang et al. 2005). MiR164 in Arabidopsis thaliana indirectly affected auxin signaling by regulating the expression of $C U C 1, C U C 2$ and $N A C 1$ members of NAC domain transcription factor families, so as to improve the cold resistance of plants (Kim et al. 2009). The expression of miR319c in Arabidopsis thaliana could upregulate under cold stress, but drought, salt and ABA stress had no effect on it (Sunkar and Zhu 2004). miR393 in Arabidopsis was stronly induced by cold, dehydration, $\mathrm{NaCl}$, and $\mathrm{ABA}$ treatments, but miR389a.1 was inhibited by these stress treatments (Zhang, 2015). At the booting stage in rice, miR1425 was inhibited under cold stress in the spikelet of Oryza sativa, resulting in the up-regulation of the expression of the target gene $R f-1$, which encoding PPR (pentatricopeptide repeat) protein. PPR protein can enhance cold tolerance by promoting cell division and increasing the number of pollen grains ( $\mathrm{Lu}$ et al. 2008). The expression of miR397 did not change under cold stress. However, miR397b. 2 of miR397 family participated in response to heat stress by inducing the expression of L-ascorbate oxidase (Jeong et al. 2011). In Arabidopsis thaliana, the expression of miR398 could be induced and its target genes CSD1, CSD2 and CCS be inhibited under heat stress (Guan et al. 2013).

In recent years, with the development of high-throughput sequencing (HTS) technology, and the combination of transcriptomics, cloning and sequencing of small RNAs, the identification of miRNAs has been deepened and developed, and it is possible to obtain a large number of miRNA sequence informations. For instance, under heat stress, 18 novel miRNAs and 41 conserved 
111

112

113

miRNA families were isolated in Brassica campestris (Ahmed et al. 2019). Zhu et al. (2019) identified 113 known miRNAs and 26 novel miRNAs in Medicago trumatida under heat stress conditions, and 202 miRNAs were ascertained in wheat (Ravichandran et al. 2019). Under drought stress, 104 upregulated and 27 downregulated miRNAs were identified in Populus euphratica (Li et al. 2011b). A total of 163 (33 known and 130 novels) miRNAs were explored as significantly differentially expressed small RNA libriries between tomato and Lycium (Goji Berry) (Khaldun et al. 2016). Moreover, the overexpression, or knock-in of transcribed MIR gene sequences has been proved to be involved in stress responses in different plant species (Basso et al. 2019), and genome-editing technologies based on the CRISPR/Cas9 or CRISPR/Cpf1 system have revealed new insights into miRNA fine-tuning (Tang et al. 2017; Zhou et al. 2017). However, the difference of miRNA expression at boostiing stage between two different rice varieties such as 9311 and N22 under heat stress is still unclear. In this study, the rice 9311 and N22 at booting stage were used matierial (heat treatment for $0,3,6$, and $12 \mathrm{~h}$, respectively), and eight small RNA libriries were construceted and deep sequenced. This study enriches the number of heat-responsive miRNAs and lays a foundation for the elucidation of the miRNA-mediated regulatory mechanism in rice at elevated temperatures.

\section{Materials and Methods}

\section{Plant materials and heat stress treatments}

The seeds of indica cultivar 9311, a high yield, eating quality, and heat-sensitive upland cultivar (Qiao et al. 2016), and Nagina 22 (N22), a popular drought- and heat-tolerant upland cultivar (Sevanthi et al. 2018), were kindly provided by State Key Laboratory of Hybrid Rice, Hunan Hybrid Rice Research Center. The seedings of two rice varieties 9311 and Nagina 22 were 
133

134

grown in greenhouse at $28{ }^{\circ} \mathrm{C}$ under a $14 \mathrm{~h}$ day $/ 10 \mathrm{~h}$ night cycle. The 40 -old-day seedlings were planted in the experimental field of the garden of Hunan Agricultural University with row spacing of $20 \mathrm{~cm}$, and plant spacing of $14 \mathrm{~cm}$. The seedlings were planted in single plant with 10 plants in each row. Field water and fertilizer management is the same as conventional field, and timely pest control. About 10 days before the rice booting stage, the rice seeding with good growth and similar characters were moved into the plastic pots, and three plants were planted in each plastic pots. When the first panicle length on the main panicle of each rice plant was about $3 \mathrm{~cm}$, we began to carry out the heat stress treatments. The plastic pots with rice plants were transferred into the man-made climate equipment at relative humidity $85 \%$ under heat stress treatment for $0,3,6$, and $12 \mathrm{~h}$ at $42^{\circ} \mathrm{C}$. The spikelet of rice were cut after heat stress at different time points, respectively. The whole samples were immediately frozen in liquid nitrogen, and the N22 sequencing samples were labeled as $\mathrm{S} 01, \mathrm{~S} 02, \mathrm{~S} 03$, and $\mathrm{S} 04$, and that 9311 were labeled as $\mathrm{S} 05$, S06, S07, and S08.

\section{Small RNA library consturction and high-throughput sequenceing}

Total RNA was extracted from S01, S02, S03, S04, S05, S06, S07 and S08 with TRIzol regent (Invitrogen, USA) following the manufacturer's instructions. Eight small RNA libraries were constructed according to previously reported procedures (Hafner et al. 2008). Briefly, small RNA fractions of $18-30 \mathrm{nt}$ isolated and purified by $15 \%$ denaturing polyacrylamide gel electrophoresis were ligated with specialized adaptors to the 5' and 3' ends (Illumina) using T4 RNA ligase. They were then reverse transcribed to cDNA using SuperScript II Reverse Transcriptase (Invitrogen), followed by PCR amplification. The final PCR products were purified and subjected to deep sequencing using Solexa sequencer (Illumina) HiSeq2500 at the Biomarker Technologies 
155

156

157

(BioMark), Beijing, China. The high-throughput sequence data are deposited in the NCBI Sequence Read Archive (SRA, https://submit.ncbi.nlm.nih.gov/subs/sra/) with accession number

PRJNA668032.

\section{Sequence analysis and identification of miRNAs}

The raw sequences were removed adaptor sequences, low-quality sequences, low-complexity regions, and shorter than $18 \mathrm{nt}$ or longer than $30 \mathrm{nt}$. Unique sequences were retained with count numbers of the individual sequence reads. For the indentification of known miRNAs, Firstly, we mapped the clean reads to miRBase22.1 (http://www.mirbase.org/) and Rfam (https://rfam.org/) databases, and filter out rRNA, tRNA, snRNA, snoRNA and repetitive sequences by miRDeep2 software (Mackowiak. 2011). Then, we mapped these un-annotated reads to the reference genomes of rice to obtain mapped reads, which can be used for further analysis. For the indentification criteria of known miRNAs, Firstly, we aligned the mapped reads of 9311 and N22 with the mature miRNA sequences of all plants in miRBase22.1 (http://www.mirbase. org/), respectively, and allowed up to three bases mismatche in interspecies. Then, the remaining reads were align with the published mature miRNA sequences in rice, such as miRBase 22.1 (http://www.mirbase. org/), and PMRD (http://bioinformatics.cau.edu.cn/PMRD/) database, and the mismatch was not allowed because of the intraspecies alignment. The remaining sequences were used to search for novel miRNAs in rice.

To study novel miRNA candidates, the remaining small RNAs were predicted by miRDeep2 software (Mackowiak. 2011). Rice genome sequence data from NCBI Genbank (http://www. ncbi.nlm.nih.gov/genbank/) and Rfam (10.1) (http://www.sanger. ac.uk/resources/databases/rfam. html) databases, and the collected rice expressed sequence tag were as a source genome. The 
177

matched sequences including the cluster unions and about 200-bp flanking sequences were defined as potential miRNAs, and those sequences are potentially related to a set of acceptable miRNA precursor sequences and the cluster unions were likely to be novel miRNA families (Chen et al. 2015). Their secondary structures were predicted by Mfold with miRNA precursor sequences (Zuker et al. 2003). The criteria for novel miRNAs should satisfy the following (Qiao et al. 2016; Zhang et al. 2006): (1) a mature sequence localized in one arm of the stem-loop structure and between 18 and $24 \mathrm{nt}$; (2) the pre-miRNA sequence folded into an appropriate stem-loop hairpin secondary structure; (3) the MFE of secondary structures $\leq-20 \mathrm{kcal} / \mathrm{mol}$; and (4) the MFE of secondary structures $\geq 0.85$.

\section{Identification of differentially expressed miRNA and functional analysis of their targets}

The counts of identified miRNAs in eight libraries were normalized as transcripts per million (TPM) according to the formula: Thenormalization formulais: normalized expression = mapped read count / total reads $\times 1,000,000$ (Zhou et al. 2010). The normalized values of miRNAs with abundance of zero were modified to 0.01 for further analysis. The remaining normalized reads were used to calculate the p-value and the change in expression abundance. miRNAs with $\log 2$ (fold change) $\geq 1$ or $\log 2$ (fold change) $\leq-1$, and $P<0.05$ were considered to be significantly differently expressed. Fold change $=\mid$ TPM ratio $\mid$ (treatment $/$ control) (Sun et al. 2015; Li et al. 2011).

Targets of the miRNAs among the known rice ORFs were predicated with two software packages, Mireap (http://sourceforge.net/projects/mireap), psRNATarget (http://plantgrn.noble. org/pRNATarget/) (Dai et al. 2018), and psRobot (https://tools4mirs.org/software/target_ prediction/psrobot/) (Srivastava et al. 2013). DAVID website (https://david.ncifcrf.gov/) was used 
199

200

for enrichment analysis of biological processes in Gene Ontology (Jiao et al. 2012). KEGG pathway analysis was carried out on KEGG website (http://www.genome. jp/kegg/) . The String website (https: //string. Embl.de/) was used to predict protein interactions, and the network regulation was constructed by Cytoscape software (Kohl et al. 2011).

\section{Validation of miRNA expression using qRT-PCR}

Real-time quantification of miRNAs by stem-loop RT-PCR was performed as described by Chen et al. (2005) and Varkonyi-Gasic et al.(2007). Stem-loop primers binds to specific miRNA at the $3^{\prime}$ region owing to the precision conferred by the primer with the exact reverse complement of six nucleotides corresponding to the 3' end of each miRNA sequence. Quantitative RT-PCR of miRNA includes two steps, and the detail steps were performed as described by Chen et al.(2015).

Real-time PCR was carried out using the SuperReal PreMix Color SYBR Green kit (TIANGEN,

China), following the manufacturer's instructions, on a LightCycler 480 machine (Roche, USA).

The U6 and actin were used as reference genes for miRNAs and their target genes, respectively. All reactions were run in triplicate. Primers used to amplify the selected genes are listed in S5.

\section{Results}

\section{Overview of transcriptome and small RNA sequencing in rice}

In order to analyze heat-responsive miRNAs between N22 and 9311 rice cultivars, we constructed eight small RNA libraries from heat treatment for $0 \mathrm{~h}, 3 \mathrm{~h}, 6 \mathrm{~h}$, and $12 \mathrm{~h}$ at booting stage in rice, respectively (see Methods for details), and sequenced them with the high-throughput Illumina Solexa system. A total of 21,329,535 and 21,406,932 raw reads were generated from rice 9311 and N22 libraries, respectively (Table 1). After removing the adaptor sequences, low quality sequences as well as sequences shorter than $18 \mathrm{nt}$, or longer than $30 \mathrm{nt}$, we obtained a large 
221

222

number of high-quality clean reads of length $18-30 \mathrm{nt}$, such as 17,157,478 (80.44\%) in 9311 rice cultivars, and 17,874,788 (83.50 \%) in N22 rice cultivars (Table 1 and Fig.1 C).

The small RNA size distribution in both rice 9311 and N22 libraries were summarized in Fig. 1A, B. The most abundant small RNAs ranged from 19 to $25 \mathrm{nt}$, and the $24 \mathrm{nt}$ small RNAs represented the most frequent length (51.08 \% in 9311, and $49.62 \%$ in N22 library, respectively).

The unique sequences obtained from rice 9311 and N22 were mapped to the rice genome (http://rapdb.dna.affrc.go.jp/download/irgsp1.html). Clear reads matching known rice scRNA, snRNA, rRNA and tRNA, and repeats associated small RNAs as well as exons and introns annotated by BLAST with Pfam10.1 (http://rfam.sanger.ac.uk), were removed. In this study, the total amount of rRNA was $19.40 \%$ in rice 9311 and $15.70 \%$ in rice N22. Note that rRNA made up less than $40 \%$ of both small RNAs, indicating that our RNA libraries were of high quality (Fig. 1D). The remaining small RNA reads were compared to the rice miRNAs in miRBase (release 22) (http://www.mirbase.org/).

Furthermore, these small RNAs were annotated into eight different categories (Table 1). Of these, 508,075 (2.93\%), 439,588 (2.80\%), 527,427 (3.17), and 609,962 (3.33\%) unique small RNAs were annotated as miRNAs in rice 9311 libraries, and 50,3871 (2.70\%), 635,841 (3.50\%), 484,141 (2.87\%), and 549,972 (3.20\%) in rice N22 libraries, respectively. Additionally, a predominant proportion of unique sequences ( $>90 \%$ in eight libraries) were unannotated small RNAs, suggesting a broad existence of novel miRNAs in rice.

\section{Identification of known miRNAs in rice}

In this study, we revealed 464 known miRNAs belong to 246 families using computational algorithms (S1). A total of 55 miRNA families belonging to 28 conserved miRNA families and 27 
243

244

non-conserved miRNA families were listed in both rice 9311 and N22 libraries, and the diversity of rice miRNA families could be determined from their number of members (Fig. 2A). As shown, most of the conserved miRNA families had members of no less than two. Of these, the miR812 and miR166 families were the largest families with 18 and 17 members, respectively. Followed by miR2118 and miR395 with 13 members, respectively. However, the conserved miRNA families such as miR394 had only one member. Moreover, most of the non-conserved miRNA families contained only one member, but seven miRNA families, namely, miR1846, miR1862, miR2871, miR444, miR5148, miR815, and miR818 had more than two members (Fig. 2A).

The number of miRNA reads in rice 9311 and N22 libraries was highly variable ranging from 0 to 395865 , and was exploited as the indicator for assessing miRNA expression level (Fig. 2B and S1). miR166 presented the highest expression abundance with 395,865 and 312,846 copies in rice $9311(42.80 \%)$ and N22 (36.30 \%) libraries, respectively. Followed by conserved miR396 also exhibited extraordinarily high abundance in both rice varieties with a total reads more than 100,000, while some other miRNA families (miR159, miR162, miR167, miR168, miR171, miR1862, miR2118, miR319, miR812 and miR820) were moderately expressed with a total reads ranging from 10,000 to 100,000 (Fig. $2 \mathrm{~B}$ and S1). However, several miRNA families including miR1856, miR2872, miR5529, miR5804, and miR6247 were detected to be expressed at an extremely low level in both rice varieties libraries (Fig. 2B and S1). Furthermore, a significant distinction in expression abundance was also observed among different members in a certain miRNA family. For example, in the miR166 family, the read number of miR1661-3p were 178489 and 148465 in rice 9311 and N22 libriries, respectively, while miR166i-5p had only 13 and 10 copies in rice 9311 and N22 libriries, respectively. This vast expression span among different 
members within a family suggested the precise expression of miRNAs under certain conditions.

\section{Identification of novel miRNA candidates}

Novel miRNA candidates were selected on the basis of the the recent annotated criteria of novel miRNAs (Meyers et al. 2008). 469 small RNA unique sequences belonging to 123 miRNA families were identified as putative novel miRNAs base on their precursor sequences and MFE1 calculation (S2). Using their prececursor sequences, their stem-loop hairpin secondary structure were predicted. The novel mature miRNAs displayed a main length distribution ranging from 21 nt to $24 \mathrm{nt}$, and $24 \mathrm{nt}$ miRNAs accounted for the highest proportion (65.04\%). In addition, The precursor lengths of 123 novel miRNAs ranged from 50 to $124 \mathrm{nt}$ with an average length of 76.56 nt (S2). MicroRNA sequences were generally located on each arm of the stem-loop hairpin structure (5'arm, $53.65 \%$, 3'arm, $46.35 \%$ ). MFEI is a good criteria for identifying non-coding and coding RNA because of the sequence is more likely to be miRNA when the MFEI is more than 0.85 , and no mRNAs, tRNAs, or rRNAs had more MFEI higher than 0.85 (Zhang et al. 2006). In this study, the identified pre-miRNAs had a minimum free energy index (MFEI) ranging from 0. 88 to 28.56 with an average of 5.13. Base on the MFEI values, there were $37,18,17$, and 2 novel miRNAs were indentified in Triticum aestivum, Oryza sativa, Zea mays, and Sorghum bicolor, respectively (Kumar et al. 2015). The identified pre-miRNAs had a minimum free energy (MFE) ranging from $-66.00 \mathrm{cal} / \mathrm{mol}$ to $-20.10 \mathrm{kcal} / \mathrm{mol}$ and an average of $-34.31 \mathrm{kcal} / \mathrm{mol}$, which is consistent with previous reports indicating that a low MFE is characteristic of miRNA precursors (Bonnet et al. 2004).

\section{Expression profiling of differentially expressed miRNAs}

To identify the differentially expressed miRNAs under heat stress, the analysis of differential 
287

288

289

290

291

292

293

294

295

296

297

expression patterns was performed using rice 9311 libraries as control (N22 VS 9311). A total of 90 differential expressed miRNAs were obtained, and their expression alterations were showed in Fig. 3. Among them, 54 miRNAs (39 known and 15 novel ones) were upregulated (Fig. 3A), and 36 miRNAs (24 known and 12 novel ones) were downregulated (Fig. 3B) by heat stress. In Fig. 3A, the upregulated miRNAs included type I, II, and III. The expression level of 22 miRNA members in type I were highly at $0,3,6$ and $12 \mathrm{~h}$ under heat stress except osa-mir1432-5p and novel_miR_117 in both rice 9311 and N22, suggesting that these miRNAs might have similar expression patterns after heat exposure. Type II was composed of 14 differentially expressed miRNAs, all of these miRNAs expression in N22, especially novel_miR_66 and osa-miR2877, were significantly higher than that in 9311. There were 18 differentially expressed miRNAs in type III, the expression level of these miRNAs were relatively lower in both rice 9311 and N22, but the expresison of novel_miR_112 and osa-miR399b were significantly higher in N22 than that in 9311. In Fig. 3B, the downregulated miRNAs included type I and II. The type I contained 24 differentially expressed miRNAs, all of these miRNAs were highly expressed in 9311, and the expression of novel_miR_9, osa-miR2880, osa-miR1429a-3p and osa-miR1863c in 9311 was significantly higher than that in N22. Type II was composed of 12 differentially expressed miRNAs, and the expression of these miRNAs were relatively lower in both rice 9311 and N22, but the expresison of osa-miR3979-3p was significantly higher in 9311 than that in N22.

In order to explore the difference of miRNA expression at different time under heat stress in rice, the rice 9311 and N22 heat stress treatment for $0 \mathrm{~h}$ were used as control, then we compared the libraries of heat stress treatment for $3 \mathrm{~h}, 6 \mathrm{~h}$ and $12 \mathrm{~h}$ with that of $0 \mathrm{~h}$, respectively $(3 \mathrm{~h}$ VS $0 \mathrm{~h}$, $6 \mathrm{~h}$ VS $0 \mathrm{~h}$, and $12 \mathrm{~h}$ VS $0 \mathrm{~h}$ ). The results showned that there were 11 upregulated differentially 
expressed miRNAs (Fig. 4A-I and S3) and 14 downregulated miRNAs (Fig. 4A-II and S3) at $3 \mathrm{~h}$, $6 \mathrm{~h}$ and $12 \mathrm{~h}$ under heat stress in rice 9311 . There were three upregulated differentially expressed miRNAs (Fig. 4B-I and S4) and 16 downregulated miRNAs (Fig. 4B-II and S4) at $3 \mathrm{~h}, 6 \mathrm{~h}$ and 12 $\mathrm{h}$ under heat stress in rice N22. The results showed that the number of upregulated miRNAs was less than that of downregulated miRNAs under heat stress.

The Log2 (fold change) values which were difference multiples of 90 differentially expressed miRNAs in Fig. 4 were used to plot, of these, the average of absolute values of 63 known (Fig. 4C) and 27 novel miRNAs (Fig. 4D) were 1.85 and 2.10, respectively. Osa-miR1432-5p, osa-miR3979-3p, and novel_miR_117 were markedly differentially expressed with an absolute value of $\log 2$ (foldchange) $>4$ (Fig. 4C, D). Further analysis also indicated that different members in a certain miRNA family might have similar or disparate expression patterns under heat stress. For example, compareed with 9311, osa-miR2118o was significantly upregulated, whereas osa-miR2118q was downregulated in N22, providing further evidence on the complexity of miRNA regulatory roles.

\section{Target gene prediction of differentally expressed miRNAs}

Predicting the target genes of miRNAs would be essential for better understanding of the biological functions for these heat-responsive miRNAs. As a result, 2773 genes were predicted to be targets for 90 heat-responsive miRNAs in N22 VS 9311. All target genes were successfully classified into three GO ontologies using DAVID website, including molecular functions, cellular components and biological processes (Fig. 5A). As shown, the main terms were "DNA binding" (GO:0003677), "transcription factor activity" (GO:0003700), “metal ion binding” (GO:0046872), and "copper ion binding" (GO:0005507) in the molecular functions. For their cellular component 
331

332

category, the "nucleus" (GO:0005634) was the most abundant subcategories. The predominant terms implicated in biological processes were "tanscription" (GO:0006351), "regulation of transcription” (GO:0006355), “developmental process involved in reproduction” (GO:0003006), and "phenylpropanoid metabolic process" (GO:0009698).

Many of the predicted target genes were stress-related TFs, including auxin response factor (ARF) family, Zinc finge GATA-type domain containing proteins (GATAs), myb domain proteins (MYBs), PETALA2 (AP2), SPB-like proteins (SPLs), NAC domain-containing proteins (NACs), Zinc finger C2H2-type domain containing protein (C2H2), bZIP, and WRKY. Moreover, several target genes encoding important enzymes or functional proteins playing roles in diverse metabolic pathways, such as VQ domain containing proteins (VQs), Zinc finger B-box domain containing proteins (BBXs), and PPR repeat domain containing proteins (PPRs), were also identified. By annotation of targets, a few transcripts were found likely o participate in plant abiotic stress responses. For instance, miR398 target was superoxide dismutase 2 (CSD2), which was related to heat stress (Guan et al. 2013). DnaJ domain containing protein (Hsp40 or J protein) were involved in response to plant development and abiotic stress (Luo et al. 2019). A set of evidences also supported the involvement of miR164 in stress responses, regulating the miRNA-mediated cleavage of NAC (Puranik et al. 2012).

The KEGG pathway analysis results showed that significant enriched pathways targeted by miRNA in spikelet tissue of rice involved in the four aspect (Fig. 5B), including plant hormone signal transduction pathway, phenylalanine metabolism pathway, photosynthesis - antenna proteins pathway, and ubiquinone and other terpenoid - quinone biosynthesis pathway. In this study, based on plant hormone signal transduction pathway, we analyzed the regulatory 
353

354

355

relationship between miRNAs and their target genes at the booting stage in rice under heat stress

(Fig. 5C). It was showed that there were 22 target genes (4.78\%) interacting in plant hormone signal transduction, which are regulated by nine differentally expressed miRNAs. In this regulated network with 22 nodes and 30 interactions, the miRNA with the largest number of target genes is novel_miR_10, which regulated the expression of 14 target genes. The target proteins with the largest number of interacting proteins or miRNAs were ethylene-insensitive protein (EIN2, Os07g0155600; OsEIL1, Os03g0324200), ethylene receptor (ERS1, Os03g0701700), Jasmonic acid (JA) signalling repressor (EG2/TIFY3, Os04g0653000), Serine/threonine protein kinase (SAPK4, Os01g0869900), and b-ZIP transcription factor (bZIP62, Os07g0686100). It has been reported that these target genes also play an important role in plant growth and response to environment stresses (Cai et al. 2014; Diédhiou et al. 2008; Yang et al. 2019; Ye et al. 2009).

\section{qRT-PCR validation of miRNAs and target genes}

To verify the results of deep sequencing and detect the dynamic expression profiles of heat-responsive miRNAs at different time-course of heat treatment $(0,3,6$, and $12 \mathrm{~h})$, the expression of 10 main differentially expressed miRNAs were analyzed with qRT-PCR (Fig. 6). As expected, the obtained data suggested that all examined miRNAs shared a coincidental expression change between small RNA sequencing and qRT-PCR, but the expression levels (or exprssion pattern) of the same miRNA in both rice 9311 and N22 were different. For known miRNAs, the expression of downregulated miR156f-5p and miR164c fell at first then abruptly increased at $6 \mathrm{~h}$, and again gradually decreased to low level at $12 \mathrm{~h}$. But miR397a, miR398b and miR399d showed an opposite expression pattern, which upregulated at first then abruptly decreased at $6 \mathrm{~h}$, and again gradually increased to high level at $12 \mathrm{~h}$. miR166k-5p had a downregulated expression trend in 
375

both rice 9311 and N22 during the whole process of heat stress. With the extension of heating stress time, miR408-3p and miR528-5p shared a similar expression pattern in both 9311 and N22, and their expression first upregulated at $3 \mathrm{~h}$, and downregulated at $12 \mathrm{~h}$. miR5496 showed an upregulated expression trend, and the relative expession of miR5496 was higher in 9311 than that of N22. For the novel miRNAs, novel_miR_15 expression increased until $12 \mathrm{~h}$ under heat stress in 9311, but there was a lower relative expression level in N22.

Furthermore, the expression patterns of ten corresponding target genes, namely jasmonic acid (JA) signalling repressor (OsEG2 targeted by miR156f-5p), NAC transcription factor 60 (OsNAC60 targeted by miR164c), ethylene-insensitive protein 2 (OsEIN2 targeted by miR166k-5p), laccase precursor protein (OsLAC4 targeted by miR397a), superoxide dismutase gene (OsCSD2 targeted by miR398b), ubiquitin-conjugating E2 enzyme (OsPHO2 targeted by miR399d), auxin-responsive Aux/IAA gene (OsIAA6 targeted by miR408-3p), cupredoxin domain containing protein (OsUCL23 targeted by miR528-5p), ethylene-insensitive3-like 1 protein (OsEIL1 targeted by miR5496), and ethylene receptor (OsETR4 targeted by novel_miR_15), were also examined to confirm the dynamic correlation between the miRNAs and their target genes under heat stress. The results showed an approximately negative correlation between the expression of miRNAs and their corresponding targets (Fig. 6). For instance, the heat-stressed downregulation of miR156f-5p and miR166k-5p led to upregulation expression of $O s E G 2$ and OsEIN2 genes, respectively. However, the expression of OsCSD2, OsIAA6, and OsEIL1 were found to be restrained by upregulated miR398b, miR408-3P and miR5496 in the early time of heat stress, respectively.

\section{Discussion}


397

398

399

400

401

402

403

404

405

406

407

408

409

410

411

412

413

\section{High-throughput sequencing of small RNA in spikelet of rice}

In rencent years, many studies have uncovered the complexity of miRNAs in regulation of plants in responses to heat stress using high-throughput sequencing technology (Ahmed et al. 2019; Ravichandran et al. 2019). As one of the most important crops, rice plant is significantly affected by high temperature stress. The previous studies have demonstrated that miRNAs play key roles in heat tolerance at the seedling and flowering stage in rice (Jeong et al. 2011; Puranik et al. 2012).

However, genome-wide analysisof rice heat stress-regulated miRNAs has not been conducted at the booting stage, which is very important for rice yield and is the most sensitive stage to heat stress. In this study, two parallel small RNA profiling experiments were performed at the booting stage using High-throughput sequencing in rice 9311 and N22. The small RNA expression were monitored at $0,3,6$, and $12 \mathrm{~h}$. Overall, $17157478(88.044 \%)$ and $17874788(83.505 \%)$ clear reads were obtained, and the total rRNA in the clear reads were $19.40 \%$ and $15.70 \%(<40 \%)$ in rice 9311 and N22, respectively (Table 1 and Fig. 1C, D). This explained that the results of sequencing in this study can cover the whole small RNA sequence, and the quality of small RNA sequences were good, which can meet the basic requirements of research and analysis. In addition, the length distribution of small RNA sequences obtained from two rice varieties were analyzed, about $90 \%$ of the sequences ranged from 19 to $25 \mathrm{nt}$, of which $24 \mathrm{nt}$ was the main sequence, followed by $21 \mathrm{nt}$ and $23 \mathrm{nt}$ (Fig. 1A, B). The distribution of these sequences length were consistent with the previous analysis in Eucommia ulmoides and cotton (Wang et al. 2016; Xie et al. 2015).

\section{Conserved and non-conserved miRNAs in rice under heat stress}

It has been reported that there were differences in miRNA family members and expression 
419

420

421

422

423

424

425

427

abundance in plants (Cai et al. 2014). Compared with non-conserved miRNAs family, conserved miRNAs family generally have higher expression abundance and more miRNA family members. For example, miR159, miR166 and miR396 family members have the highest expression abundance in most plants (Dan et al. 2018; Zhou et al. 2018). Similarly, this study showed that miR159, miR166, miR167 and miR396 family members in rice 9311 and N22 also had the highest expression abundance under heat stress (Fig. 2B), these conserved miRNAs may play a variety of regulatory roles in plant physiological functions. For example, highly conserved miR159 affects plant development, flowering time and seed shape by regulating the expression of MYB TFs (Palatnik et al. 2007). The miR166 family targets homeodomain-leucine zipper (HD-Zip) TFs, which involved in response to heat stress in Arabidopsis (Zhong et al. 2013). The miR396 family was found to involved in cell proliferation and leaf development by targeting the growth-regulating factor (GRF) and basic helix loop helix (bHLH) TFs (Debernardi et al. 2013).

On the contrary, some non-conserved miRNAs, such as miR1868, miR3979, miR530, miR5504 and miR6249, had low expression abundance and few family members. The reasons were that these miRNAs had no homologous genes or did not exist in other plants, or their functions were not yet clear in other plants (Liu et al. 2013).

\section{Heat-responsive miRNAs in rice under heat stress}

The expression of different members of the same miRNA family were also different. In present study, 90 differentially expressed miRNAs were indentified with 9311 libraries as control, of which 54 miRNAs expression were upregulated, and 36 miRNAs were downregulated (Fig. 3A, B). Among miR167 family members, the expression level of osa-miR167h-3p was the highest, followed by osa-miR167e-3p, and osa-miR167d-3p was the lowest. In miR396 family members, 
441 the expression level of osa-miR396e-5p was the highest, followed by osa-miR396d, and 442 osa-miR396f-3p was the lowest. Among miR399 family members, the expression level of 443 osa-miR399d was the highest, followed by osa-miR399j, and osa-miR399b was the lowest.

444 MiR156a, miR156f, miR156g and miR156h have been validated in regulation of Oryza sativa 445 (Chen et al. 2012), Triticum aestivum (Xin et al. 2010), and Arabidopsis thaliana (Kim et al. 2012) in responses to heat stress. MiR164, miR166 and miR167 in switchgrass and barley were involved 447 in the regulation network under heat stress (Hivrale et al. 2016; Kruszka et al. 2014). MiR396, miR397, miR398, miR399 and miR408, in sunflower (Giacomelli et al. 2012), cassava (Ballén-Taborda et al. 2013), Arabidopsis thaliana (Kim et al. 2012), rice (Chen et al. 2012), and wheat (Xin et al. 2010), respectively, were widely reported to play important roles in heat stress responses. These results were consistented with this study. In recent years, miRNAs have been extensively studied in many other species in response to heat stress. 790 putative miRNAs to be involved in pollen development or heat stress response in Solanum lycopersicum (Keller et al. 2020). Liu et al. (2020) analysis of 523 miRNAs from 120 sRNA libraries, of which osa-miR160f-5p and ata-miR396c-5p were significantly down-regulated in DBA Aurora under water-deficit plus heat stress. High temperature stress significantly altered the expression of miRNAs, after $6 \mathrm{~h}$ of high temperature treatment, the expression levels of miR159, miR162, miR395, miR393 and miR408 were extreme significantly higher than that in the un-treated 459 sweetpotato leaves, but miR160 was decreased (Yu et al. 2020). Ahmed et al. (2020) reported that bra-miR160a, bra-miR172c-3p, bra-miR1885a, bra-miR571f8, bra-miR5726, bra-miR390-5p and bra-miR400-5p were significantly up-regulated and bra-miR156e-3p, bra-miR157a, bra-miR398- 
heat-sensitive flowering Chinese cabbage. MiR408-3p and miR9774 were significantly up-regulated in heat-tolerant Festuca arundinacea (PI578718), but down-regulated in heatsensitive Festuca arundinacea (PI234881) (Li et al. 2017). All these findings indicate that miRNAs play important roles in regulating heat stress in different species.

\section{miRNAs play crucial roles in heat stress by regulating the target genes in rice}

Differentially expressed miRNAs have the potential to regulate target transcripts to exert their functions in rice (Kozomara et al. 2019). In this study, Gene Ontology enrichment and KEGG pathway analysis were carried out on target genes of 90 differentially expressed miRNAs (56 upregulated miRNAs, 34 downregulated miRNAs). The results showed that target genes of these differentially expressed miRNAs were significantly enriched in plant hormone signal transduction pathway (Fig. 5B). Hormones were important regulators of plant growth and development, and miRNAs could affect hormone signaling pathways (Curaba et al. 2014) and involve in many different developmental processes, including phase transition, organogenesis, cell division and stress response, by inhibiting target genes (Voinnet et al. 2009). miRNAs perform regulatory functions in plant hormone signaling pathways in two ways: the first way is that miRNA inhibits the expression of target genes, which directly regulates hormone response as a key factor in hormone signaling pathway; the second way is that the interaction, between target genes and hormone signaling related genes, cause the change of plant sensitivity to hormone signal, and participate in the regulation of plant development and the response to environmental signals (Srivastava et al. 2013; Achard et al. 2004). Nine differentially expressed miRNAs and 22 target genes involved in the regulation of plant hormone signal transduction pathway in present study

(Fig. 5C). The qRT-PCR validation of expression levels of ten miRNAs and their target genes 
485

486

487

488

489

490

491

492

493

494

495

496

497

498

499

500

501

502

503

504

505

506

were generally consistent with the sequencing results, showing the accuracy of the sequencing result (Fig. 6). The expression of osa-miR398b increased first and then decreased, and it negatively regulated the expression of $O S C S D 2$, this result was consistent with the previous analysis in Arabidopsis thaliana (Guan et al. 2013). osa-miR397a and osa-miR399d positively regulate the expression of their target genes OsLAC4 and OSPHO2 respectively, which is consistent with the results reported by Gahlaut et al.(Gahlaut et al. 2018). osa-miR408-3p and osa-miR5496 were induced by high temperature, and their expression level were upregulated after heat stress treatment for $3 \mathrm{~h}$. The target genes OsIAA6 and OsEIL1 were negatively regulated by osa-miR408-3p and osa-miR5496, respectively.

Previous studies have shown that miRNA could participate in response to high temperature stress through regulating hormone response pathway in plants. Under heat stress, miR160, miR167, miR390 and miR393 were involved in auxin metabolism (Goswami et al. 2014; Kruszka et al. 2014; Lin et al. 2018), and miR159 could regulate gibberellins (GA3) metabolic pathway (Li et al. 2016), and miR319 was involved in the regulation of jasmonic acid metabolic pathway (Hivrale et al. 2016). Many miRNAs were also induced by plant hormones, such as aux, which enhanced the expression of miR164, miR319 and miR390 (Guo et al. 2005; Srivastava et al. 2013; Yoon et al. 2010); abscisic acid (ABA) induced the expression of miR393 (Sunkar et al. 2004), but inhibited the expression of miR167 and miR319 (Liu et al. 2009); ethylene (ET) downregulated the expression of miR159a, miR164a/b/c and miR319/390 (Chen et al. 2012; Zuo et al. 2012), which indicated that miRNA might be regulated by hormone signal.

However, compared with the previous study at the seedling and flowing stage (Jeong et al. 2011; Puranik et al. 2012; Xin et al. 2010), we also identified different set of heat responsive miRNAs in 
507

508

509

510

511

512

513

the present study. For example, many miRNAs, such as miR156, miR164, and miR166, were upregulated by heat stress in wheat (Xin et al. 2010; Pandey et al. 2014), whereas these miRNAs were downregulated and their target genes, such as OsEG2, OsNAC and OsEIN2, were upregulated in our study. MiR397b was downregulated at the flowering stage (Jeong et al. 2011) while miR397a was induced by heat stress at the booting stage in rice. This may be due to the different expression of miRNA in different species at different growth and development stages.In summary, The study provides the first comprehensive and specific miRNA profile of heat-induced miRNAs in spikelet of rice. The identification of genotype and tissue preferential miRNAs will help in widening the knowledge base of miRNA-mediated gene regulation in rice.

\section{Acknowledgements}

We are grateful to our editors and reviewers for their helpful comments and the groups who submitted the sequencing data to the NCBI Sequence Read Archive. This work was supported by State Commission of Science Technology of China (2014BAD01B04), Hunan province university innovation platform open fund project (13K063), The Natural Science Foundation of Hunan Province (2018JJ2144), Scientific Research Foundation of Hunan Education Department (18A480), Scientific Research Foundation of Hunan University of Science and Engineering (17XKY005).

\section{Competing financial interests}

The authors declare no competing financial interests.

\section{References}

Achard, P., Herr, A., Baulcombe, D., and Harberd, N. P. 2004. Modulation of floral development by a gibberellin-regulated microRNA. Development. 131(14): 3357-3365. 
529

530

531

532

533

534

535

536

537

538

539

540

541

542

543

544

Ahmed, W., Xia, Y.S., Zhang, H., Li, R.H., Bai, G.H., Siddique, K.H.M., et al. 2019. Identification of conserved and novel miRNAs responsive to heat stress in flowering Chinese cabbage using high-throughput sequencing. Sci Rep. 9: 14922.

Ahmed, W., Li, R., Xia, Y.S., Bai, G.H., Siddique, K.H.M. Zhang, H., et al. 2020. Comparative analysis of miRNA expression profiles between heat-tolerant and heat-sensitive genotypes of flowering Chinese cabbage under heat stress using high-throughput sequencing. Genes. 11(3):264.

Allen, E., Xie, Z.X., Gustafson, A.M., and Carrington, J.C. 2005. microRNA-directed phasing during trans-acting siRNA biogenesis in plants. Cell. 121(2): 207-221.

Ballén-Taborda, C., Plata, G., Ayling, S., Rodríguez-Zapata, F., Lopez-Lavalle, L.A.B., Duitama, J., et al. 2013. Identification of Cassava MicroRNAs under abiotic stress. Int J Genomics. 5: $1-10$.

Basso, M.F., Ferreira P.C.G., Kobayashi, A.K., Harmon, F.G., Nepomuceno, A.L., Molinari, H.B.C., et al. 2019. MicroRNAs and new biotechnological tools for its modulation and improving stress tolerance in plants. Plant Biotechnol J. 17(8): 1482-1500.

Bokszczanin, K.L., and Fragkostefanakis, S. 2013. Perspectives on deciphering mechanisms underlying plant heat stress response and thermotolerance. Front Plant Sci. 4: 315.

Bonnet, E., Wuyts, J., Rouze, P., and van de Peer, Y. 2004. Evidence that microRNA precursors, unlike other non-coding RNAs, have lower folding free energies than random sequences. Bioinformatics. 20(17), 2911-2917.

Cai, Q., Yuan, Z., Chen, M.J., Yin, C.S., Luo, Z.J., Zhao, X.X. et al., 2014. Jasmonic acid regulates spikelet development in rice. Nat Commun. 5: 3476. 
551 Chen, C.F., Ridzon, D.A., Broomer, A.J., Zhou, Z.H., Lee D.H., Nguyen JL., et al. 2005.

552 Real-time quantification of microRNAs by stem-loop RT-PCR. Nucleic Acids Res. 33(20): 553 e179.

554 Chen, C.C., Fu, S.F., Norikazu, M., Yang, Y.W., Liu, Y.J., Ikeo, K., et al. 2015. Comparative 555 miRNAs analysis of Two contrasting broccoli inbred lines with divergent head-forming capacity under temperature stress. BMC Genomics. 16: 1026.

557 Chen, L., Wang, T.Z., Zhao, M.G., and Zhang, W.H. 2012. Ethylene-responsive miRNAs in roots 558 of Medicago truncatula identified by high-throughput sequencing at whole genome level. Plant 559 Sci. 184: 14-19.

Chen, X. 2012. Small RNAs in development - insights from plants. Curr Opin Genet Dev. 22(4): $361-367$.

Ciais, P., Reichstein, M., Viovy, N., Granier, A., Ogée, J., Allard, V., et al. 2005. Europewide reduction in primary productivity caused by the heat and drought in 2003. Nature. 437(7058): $529-533$.

Cui, J., You, C., and Chen, X. 2017. The evolution of microRNAs in plants. Curr Opin in Plant Biol. 35: 61-67.

Curaba, J., Singh, M.B., and Bhalla, P.L. 2014. miRNAs in the crosstalk between phytohormone signalling pathways. J Exp Bot. 65(6): 1425-1438.

Dai, X. B., Zhuang, Z.H. and Zhao, P.X. 2018. psRNATarget: A Plant Small RNA Target Analysis Server (2017 Release). Nucleic Acids Res. 46(W1): W49-W54. ethylene responsive miRNAs and their targets from newly harvested banana fruits using 

specialization of the plant miR396 regulatory network through distinct microRNA-target interactions. PLOS Genet. 8(1): e1002419.

Diédhiou, C.J., Popova, OV., Dietz, K.J., and Golldack, D. 2008. The SNF1-type serine-threonine protein kinase SAPK4 regulates stress-responsive gene expression in rice. BMC Plant Biol. 8: 49.

\section{Plant Biol. 7(5): 512-520.}

Gahlaut, V., Baranwal, V.K., and Khurana, P. 2018. miRNomes involved in imparting thermotolerance to crop plants. 3 Biotech. 8(12): 497.

Giacomelli, J.I., Weigel, D., Chan, R.L., and Manavella, P.A. 2012. Role of recently evolved miRNA regulation of sunflower HaWRKY6 in response to temperature damage. New Phytol. 195(4): 766-773.

Goswami, S., Kumar, R.R., and Rai, R.D. 2014. Heat-responsive microRNAs regulate the transcription factors and heat shock proteins in modulating thermo stability of starch biosynthesis enzymes in wheat (Triticum aestivum L. ) under the heat stress. Aust J Crop Sci. 8(5): 697-705.

Guan, Q.M., Lu, X.Y., Zeng, H.T., Zhang, Y.Y., and Zhu, J.H. 2013. Heat stress induction of miR398 triggers a regulatory loop that is critical for thermotolerance in Arabidopsis. Plant J. 74(5): 840-851.

594 Guo, H.S., Xie, Q., Fei, J.F., and Chua, N.H. 2005. MicroRNA directs mRNA cleavage of the 
595

596

597

598

599

600

601

602

603

604

605

606

607

608

609

610

611

612

613

614

615

616

transcription factor $\mathrm{NAC1}$ to downregulate auxin signals for Arabidopsis lateral root development. Plant Cell. 17(5): 1376-1386.

Hafner, M., Landgraf, P., Ludwig, J., Rice, A., Ojo, T., Lin, C., et al. 2008. Identification of microRNAs and other small regulatory RNAs using cDNA library sequencing. Methods. 44(1): $3-12$.

Hawkins, E. Fricker, T.E., Challinor, A.J., Ferro, C.A.T., Ho, C.K., and Osbome, T.M. 2013. Increasing influence of heat stress on French maize yields from the 1960s to the 2030s. Global Change Biol. 19(3): 937-947.

Hivrale, V. Zheng, Y., Puli, C.O.R., Jagadeeswaran, G.J., Gowdu, K., et al. 2016. Characterization of drought- and heat-responsive microRNAs in switchgrass. Plant Sci. 242: 214-223.

IPCC. Climate Change 2013: 2013. The Physical Science Basis. Contribution of working group I to the fifth assessment report of the intergovernmental panel on climate change. Cambridge, United Kingdom and New York, NY, USA: Cambridge University Press, 1535.

Jeong, D.H., Park, S., Zhai, J.X., Gurazada S.G.R., Paoli, E.D., Meyers, B.C., et al. 2011. Massive analysis of rice small RNAs: mechanistic implications of regulated microRNAs and variants for differential target RNA cleavage. Plant Cell. 23(12): 4185-4207.

Jiao, X.L., Sherman, B.T., Huang, D.W., Stephens, R., Baseler, MW., Lane H.C., et al. 2012. DAVID-WS: a stateful web service to facilitate gene/protein list analysis. Bioinformatics. 28(13): 1805-1806.

Keller, M., Schleiff, E., Simm, S. 2020. miRNAs involved in transcriptome remodeling during pollen development and heat stress response in Solanum lycopersicum. Sci Rep. 10(1): 10694.

Khaldun, A.B.M., Huang, W.J., Lv, H.Y., Liao, S.H., Zeng, S.H., and Wang, Y. 2016. 
617

618

619

620

621

622

623

624

625

626

627

628

629

630

631

632

633

634

635

636

637

638

Comparative Profiling of miRNAs and Target Gene Identification in Distant-Grafting between Tomato and Lycium (Goji Berry). Front in Plant Sci. 7: 1475.

Kim, J.H., Woo, H.R., Kim, J., Lim, P.O., Lee, I.C., Choi, S.H., et al. 2009. Trifurcate feed-forward regulation of age-dependent cell death involving miR164 in Arabidopsis. Science. 323(5917): 1053-1057.

Kim, J.J., Lee, J.H., Kim, W., Jung, H.S., Huijer, P., and Ahn, J.H. 2012. The miR156-SPL3 module regulates ambient temperature-responsive flowering via $F T$ in Arabidopsis thaliana. Plant Physiol. 159(1): 461-478.

Kohl, M., Wiese, S., and Warscheid, B. 2011. Cytoscape: software for visualization and analysis of biological networks. Methods Mol Biol. 696: 291-303.

Kozomara, A., Birgaoanu, M., and Griffiths-Jones, S. 2019. miRBase: from microRNA sequences to function. Nucleic Acids Res. 47(D1), D155-D162.

Kruszka, K. Pacak, A., Swida-Barteczka, A., Nuc, P., Alaba, S., Wroblewska, Z., et al. 2014. Transcriptionally and post-transcriptionally regulated microRNAs in heat stress response in barley. J Exp Bot. 65(20): 6123-6135.

Kumar, R.R., Pathak, H., Sharma, S.K., Kala, Y.K., Nirjal, M.K., Singh, G.P., et al. 2015. Novel and conserved heat-responsive microRNAs in wheat. Funct Integr Genomics. 15: 323-348.

LeComte, D.U.S. 2013. weather highlights 2012: heat, drought, and sandy. Weather Wise, 66(3): $12-19$.

Li, B., Qin, Y., Duan, H., Yin, W., and Xia, X. 2011b. Genome-wide characterization of new and drought stress responsive microRNAs in Populus euphratica. J Exp Bot. 62(11): 3765-3779.

Li, H., Wang, Y., Wang, Z., Guo, X., Wang, F., Xia, X.J., et al. 2016. Microarray and genetic 
639

640

641

642

643

644

645

646

647

648

649

650

651

652

653

654

655

656

657

658

659

660

analysis reveals that csa-miR159b plays a critical role in abscisic acid-mediated heat tolerance in grafted cucumber plants. Plant Cell Environ. 39(8): 1790-1804.

Li, H.Y., Hu, T., Amombo, E., Fu, J.M. 2017. Genome-wide identification of heat stress-responsive small RNAs in tall fescue (Festuca arundinacea) by high-throughput sequencing.

J Plant Physiol. 213: 157-165.

Li, H.Y., Dong, Y.Y., Yin, H.L., Wang, N., Yang, J., Liu, X.M., et al. 2011. Characterization of the stress associated microRNAs in Glycine max by deep sequencing. BMC Plant Biol. 11: 170.

Li, S.B., Liu, L., Zhuang, X.H., Yu, Y., Liu, X.G., Cui, X., et al. 2013. MicroRNAs inhibit the translation of target mRNAs on the endoplasmic reticulum in Arabidopsis. Cell. 153(3): $562-574$

Lin, J.S., Kuo, C.C., Yang, I.C., Tsai, W.A., Shen, Y.H., Lin, C.C., et al. 2018. MicroRNA160 modulates plant development and heat shock protein gene expression to mediate heat tolerance in Arabidopsis. Front Plant Sci. 9: 68.

Liu, H.P., Able, A.J., Able, J.A. 2020. Integrated analysis of small RNA, transcriptome, and degradome sequencing reveals the water-deficit and heat stress response network in durum wheat. Int J Mol Sci. 21(17): 6017.

Liu, N., Yang, J., Guo, S., Xu, Y. and Zhang, M. 2013. Genome-wide identification and comparative analysis of conserved and novel microRNAs in grafted watermelon by high-throughput sequencing. PloS ONE. 8(2): e57359.

Liu, P.P., Montgomery, T.A., Fahlgren, N., Kasschau, K.D., Nonogaki, H., and Carrington, J.C. 2007. Repression of auxin response factor 10 by microRNA160 is critical for seed germination and post-germination stages. Plant J. 52(1): 133-146. 
661

662

663

664

665

666

667

668

669

670

671

672

673

674

675

676

677

678

679

680

681

682

Liu Q. Zhang, Y.H., Wang, C.Y., Luo, Y.C., Huang, Q.J., Chen, S.Y., et al. 2009. Expression analysis of phytohormone-regulated microRNAs in rice, implying their regulation roles in plant hormone signaling. FEBS Lett. 583(4): 723-728.

Lobell, D.B., and Burke, M.B. 2010. On the use of statistical models to predict crop yield responses to climate change. Agr Forest Meteorol. 150(11): 1443-1452.

Lu, C. Jeong, D.H., Kulkarni, K., Pillay, M., Nobuta, K., German, R., et al. 2008. Genome-wide analysis for discovery of rice microRNAs reveals natural antisense microRNAs (nat-miRNAs). P Natl Acad Sci USA. 105(12), 4951-4956.

Luo, Y. Fang, B.H., Wang, W.P., Yang, Y., Rao, L.Q., and Zhang, C. 2019. Genome-wide analysis of the rice J-protein family: identification, genomic organization, and expression profiles under multiple stresses. 3 Biotech. 9: 358.

Mackowiak, S.D. 2011. Identification of novel and known miRNAs in deep-sequencing data with miRDeep2. Current Protocols in Bioinformatics. Chapter 12:Unit 12.10.

Meyers, B.C., Axtell, M.J., Bartel, B., Bartel, D.P., Baulcombe, D., Bowman, J.L., et al. 2008. Criteria for annotation of plant MicroRNAs. Plant Cell. 20: 3186-3190.

Palatnik, J.F., Wollmann, H., Schommer, C., Schwab, R., Boisboubier J., Rodriguez, R., et al. 2007. Sequence and expression differences underlie functional specializationof Arabidopsis microRNAs miR159 and miR319. Develemental Cell. 13(1): 115-125.

Pandey, R., Joshi, G., Bhardwaj, A.R., Agarwal, M. and Katiyar-Agarwal, S. 2014. A Comprehensive genome-wide study on tissue-specific and abiotic stress-specific miRNAs in Triticum aestivum. PLoS ONE. 9(4): e95800.

Puranik, S., Sahu, P.P., Srivastava, P.S., and Prasad, M. 2012. NAC proteins: regulation and role 
683

684

685

686

687

688

689

690

691

692

693

694

695

696

697

698

699

700

701

702

703

704

in stress tolerance. Trends Plant Sci. 17(6): 369-381.

Qiao, W.H., Qi, L., Cheng, Z.J., Su, L., Li, J., Sun, Y., et al. 2016. Development and characterization of chromosome segment substitution lines derived from Oryza rufipogon in the genetic background of O. sativa spp. indica cultivar 9311. BMC Genomics. 17: 580.

Ravichandran, S., Ragupathy, R., Edwards, T., Domaratzki, M., and Cloutier, S. 2019. MicroRNA- guided regulation of heat stress response in wheat. BMC Genomics. 20: 488.

Sevanthi A.M.V., Kandwal, P., Kale, P.B., Prakash, C., Ramkumar, M.K., Yadav, N., et al. 2018. Whole genome characterization of a few EMS-induced mutants of upland rice variety Nagina 22 reveals a staggeringly high frequency of SNPs which show high phenotypic plasticity towards the wild-type. Front Plant Sci. 9: 1179.

Srivastava, S., Srivastava, A.K., Suprasanna, P., and D'Souza, S.F. 2013. Identification and profiling of arsenic stress-induced microRNAs in Brassica juncea. J Exp Bot. 64(1): 303-315.

Sun, X.C., Xu, L., Wang, Y., Yu, R.G., Zhu, X.W., Luo, X.B., et al. 2015. Identification of novel and salt-responsive miRNAs to explore miRNA- mediated regulatory network of salt stress response in radish (Raphanus sativus L.). BMC Genomics. 16: 197.

Sunkar, R. and Zhu, J.K. 2004. Novel and stress-regulated microRNAs and other small RNAs from Arabidopsis. Plant Cell. 16(8): 2001-2019.

Tang, X., Lowder, L.G., Zhang, T., Malzahn, A.A., Zheng, X., Voytas, D.F., et al. 2017. A CRISPR-Cpf1 system for efficient genome editing and transcriptional repression in plants. Nat. Plants, 3: 17018 .

Varkonyi-Gasic, E., Wu, R., Wood, M., Walton, E.F., and Hellens, R.P. 2007. Protocol: a highly sensitive RT-PCR method for detection and quantification of microRNAs. Plant Methods. 3: 
706

Voinnet, O. 2009. Origin, biogenesis, and activity of plant microRNAs. Cell. 136(4): 669-687.

Wahid, A., and Close, T.J. 2007. Expression of dehydrins under heat stress and their relationship with water relations of sugarcane leaves. Biol Plantarum. 51(1): 104-109.

Wang, J.W., Wang, L.J., Mao, Y.B., Cai, W.J., Xue, H.W., and Chen, X.Y. 2005. Control of root cap formation by microRNA targeted auxin response factors in Arabidopsis. Plant Cell. 17(8): 2204-2216.

Wang, L., Du, H., and Wuyun, TN. 2016. Genome-Wide identification of microRNAs and their targets in the leaves and fruits of Eucommia ulmoides using high-throughput sequencing. Front Plant Sci. 7: 1632.

Wassmann, R., Jagadish, S.V.K., Heuer, S., Ismail, A., Redona, E., Serraj, R., et al. 2009. Climate change affecting rice production: the physiological and agronomic basis for possible adaptation strategies. Adv Agron. 101, 59-122.

Wu, L., Zhou, H.Y., Zhang, Q.Q., Zhang, J.G., Ni, F.R., Liu, C., et al. 2010. DNA methylation mediated by a microRNA pathway. Mol Cell. 38(3): 465-475.

Xie, F.L., Wang, Q.L., Sun, R.R., and Zhang, B.H. 2015. Deep sequencing reveals important roles of microRNAs in response to drought and salinity stress in cotton. J Exp Bot. 66(3): 789-804.

Xie, Z., Allen, E., Fahlgren, N., Calamar, A., Givan, S.A., and Carrington, J.C. 2005. Expression of Arabidopsis miRNA genes. Plant Physiol. 138: 2145-2154.

Xin, M.M., Wang, Y., Yao, Y.Y., Xie, C.J., Peng, H.R., Ni, Z.F., et al. 2010. Diverse set of microRNAs are responsive to powdery mildew infection and heat stress in wheat (Triticum aestivum L.). BMC Plant Biol. 10: 123. 
727

728

729

730

731

732

733

Yang, S.Q., Xu, K., Chen, S.J., Li, T.F., Xia, H., Chen, L., et al. 2019. A stress-responsive bZIP transcription factor OsbZIP62 improves drought and oxidative tolerance in rice. BMC Plant Biol. 19(1): 260 .

Ye, H.Y., Du, H., Tang, N., Li, X.H., and Xiong, L.Z. 2009. Identification and expression profiling analysis of TIFY family genes involved in stress and Phytohormone responses in rice. Plant Mol Biol. 71: 291-305.

Yoon, E.K., Yang, J.H., Lim, J., Kim, S.W., Kim, S.K., and Lee, W.S. 2010. Auxin regulation of the microRNA390-dependent transacting small interfering RNA pathway in Arabidopsis lateral root development. Nucleic Acids Res. 38(4): 1382-1391.

Yu, J.J., Su, D, Yang, D.J., Dong, T.T., Tang, Z.H., Li, H.M., et al. 2020. Chilling and heat stress-induced physiological changes and microRNA-related mechanism in sweetpotato (Ipomoea batatas L.). Front Plant Sci. 11: 687.

Zhang, B.H., Pan, X.P., Cox, S.B., Cobb, G.P., Anderson, T.A. 2006. Evidence that miRNAs are different from other RNAs. Cell Mol Life Sci. 63(2): 246-254.

Zhang B. H. 2015. MicroRNA: a new target for improving plant tolerance to abiotic stress. J Exp Bot. 66(7): 1749-1761.

Zhong, S.H., Liu, J.Z., Jin, H., Lin. L., Li, Q., Chen, Y., et al. 2013. Warm temperatures induce transgenerational epigenetic release of RNA silencing by inhibiting siRNA biogenesis in Arabidopsis. P Natl Acad Sci USA. 110(22): 9171-9176.

Zhou, J.P., Deng, K. J., Cheng, Y., Zhong, Z. H., Tian, L., Tang, X., et al. 2017. CRISPR/Cas9 based genome editing reveals new insights into microRNA function and regulation in rice. Front Plant Sci. 8: 1598. 
749

750

751

752

753

754

755

756

757

758

759

760

761

762

763

764

765

766

767

768

769

770

Zhou, L., Chen, J.H., Li, Z.Z., Li, X.X., Hu, X.D., Huang, Y., et al. 2010. Integrated profiling of microRNAs and mRNAs: microRNAs located on Xq27.3 associate with clear cell renal cell carcinoma. PloS ONE. 5(12): e15224.

Zhou, L., Quan, S., Xu, H., Ma, L., and Niu, J. 2018. Identification and Expression of miRNAs related to female flower induction in Walnut (Juglans regia L.). Molecules. 23(5): 1202.

Zhu, H., Zhang, Y., Tang, R.F., Qu, H.X., Duan, X.W., and Jiang, Y.M. 2019. Banana sRNAome and degradome identify microRNAs functioning in differential responses to temperature stress. BMC Genomics. 20: 33.

Zuker, M. 2003. Mfold web server for nucleic acid folding and hybridization prediction. Nucleic

Acids Res. 31(13): 3406-3415.

Zuo, J.H., Zhu, B.Z., Fu, D.Q., Zhu, Y., Ma, Y.Z., Chi, L.H., et al. 2012. Sculpting the maturation, softening and ethylene pathway: The influences of microRNAs on tomato fruits. BMC Genomics. 13: 7. 
Fig. 1. The length distribution and annotation of small RNAs from the rice 9311 and N22 libraries sequences from raw reads in rice 9311 and N22 libraries. D: The proportions of different classes of small RNAs detected in rice 9311 and N22.

Fig. 2. Distribution of member numbers (A) and abundance (B) of each known miRNA family in rice 9311 and N22 libraries under heat stress. X axis represents miRNA families, and Y axis represents member numbers $(\mathrm{A})$ and abundance $(\mathrm{B})$, respectively.

Fig. 3. Expression profiling of of differentially expressed miRNAs in N22 versus 9311 (N22 VS 9311). A, B: The expression of upregulated and downregulated differentially expressed miRNAs in N22 VS 9311. Red and green indicate high and low expression level, respectively.

Fig. 4. Expression profiling and fold change ( $\log 2)$ of of differentially expressed miRNAs under differentially expressed miRNAs in rice N22 with $0 \mathrm{~h}$ as control. Red and green indicate high and low expression level, respectively. Comparative relative expression of differentally exressed known miRNAs (C) and novel miRNAs (D) in N22 VS 9311. The miRNAs with positive fold change value are upregulated, and the ones with negative fold change value are downregulated. 
793

794

795

796

797

798

799

800

801

802

803

804

805

806

807

808

809

810

811

812

813

814

in N22 VS 9311. X axis represents -Log (PValue); Numbers represent gene counts; Blue, yellow and green represent three GO ontologies: molecular function, cellular componet and biological progress, respectively. B: Enriched KEGG pathway for target genes in N22 VS 9311. P-values range from 0 to 1 , and its less value means greater intensiveness. Top 10 pathway terms enriched by KEGG database were carried out. C: Regulatory network linking the differential expressed miRNA and their target genes in N22 VS 9311 in plant hormone signal transduction pathway, which showed in Fig. 6B. Red circular nodes represent miRNAs, green circular nodes represent target genes.

Fig. 6. The expression patterns of miRNAs and their targets in the spikelet of rice 9311 and N22 at control $(0 \mathrm{~h})$ or heat srress treatments $(3 \mathrm{~h}, 6 \mathrm{~h}$, and $12 \mathrm{~h})$. Each value was calculated as the mean of three samples \pm standard error (SE). The different letters above the bar indicate significant difference $(\mathrm{P}<0.05)$.

(1)


815

816 Table 1. Summary of eight small RNA libraries in spikelet tissue of the rice 9311 and N22

817 libraries

818

\begin{tabular}{lccccccc}
\hline Sample & $\begin{array}{l}\text { Total number } \\
\text { of raw reads }\end{array}$ & $\begin{array}{l}\text { Total number } \\
\text { of clear reads }\end{array}$ & $\begin{array}{l}\text { Number of reads } \\
\text { mapped to rice } \\
\text { genome }\end{array}$ & $\begin{array}{l}\text { Number of reads } \\
\text { mapped to } \\
\text { mature miRNA }\end{array}$ & $\begin{array}{l}\text { mapped to } \\
\text { rice } \\
\text { genome \% }\end{array}$ & $\begin{array}{l}\text { mapped to } \\
\text { mature } \\
\text { miRNA \% }\end{array}$ & $\begin{array}{l}\text { Number of } \\
\text { mature miRNAs } \\
\text { detected }\end{array}$ \\
\hline 9311-0h & 22041929 & 17730528 & 12866716 & 508075 & 72.77 & 2.93 & 293 \\
$9311-3 \mathrm{~h}$ & 19348844 & 15564210 & 11398278 & 439588 & 73.27 & 2.80 & 292 \\
$9311-6 \mathrm{~h}$ & 20633140 & 16597298 & 11930169 & 527427 & 71.73 & 3.17 & 301 \\
$9311-12 \mathrm{~h}$ & 23294227 & 18737876 & 13500388 & 609962 & 72.10 & 3.33 & 319 \\
$\mathrm{~N} 22-0 \mathrm{~h}$ & 22319091 & 18636441 & 13853548 & 503871 & 74.47 & 2.70 & 296 \\
$\mathrm{~N} 22-3 \mathrm{~h}$ & 21775169 & 18182266 & 13183213 & 635841 & 72.53 & 3.50 & 295 \\
$\mathrm{~N} 22-6 \mathrm{~h}$ & 20595992 & 17197653 & 12496489 & 484141 & 72.70 & 2.87 & 295 \\
$\mathrm{~N} 22-12 \mathrm{~h}$ & 20937475 & 17482792 & 12913480 & 549972 & 73.90 & 3.20 & 297 \\
\hline
\end{tabular}

819

820

821

822

823

824

825

826

827

828

829

830 


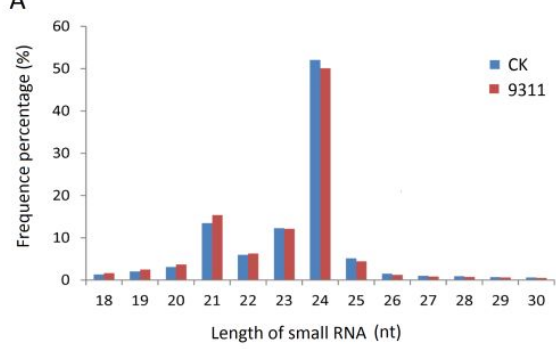

B

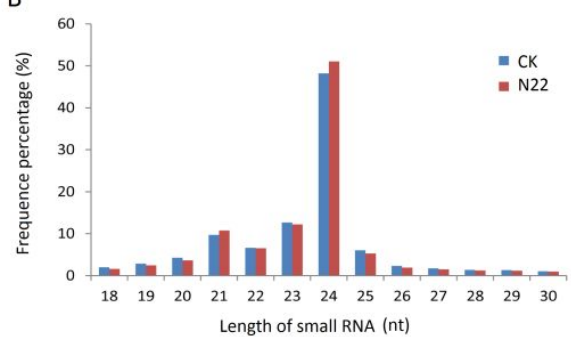

C

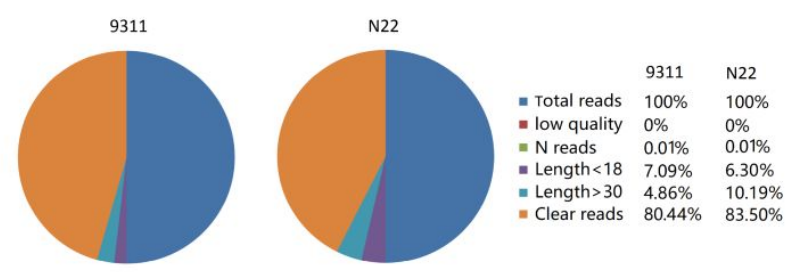

D
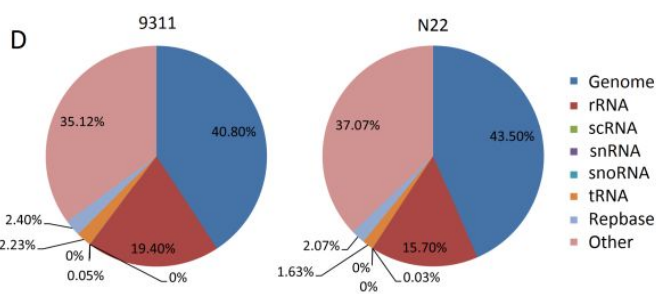

833

834

835

836

837

838

839

840

841

842

843

(c) The Author(s) or their Institution(s) 
A

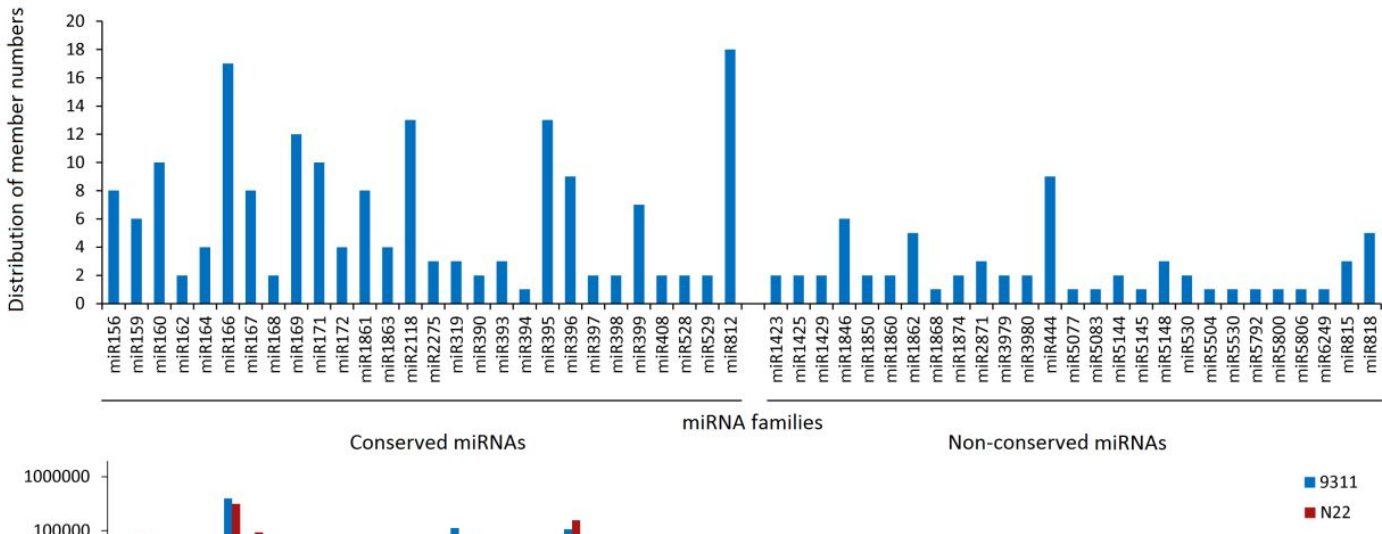


A

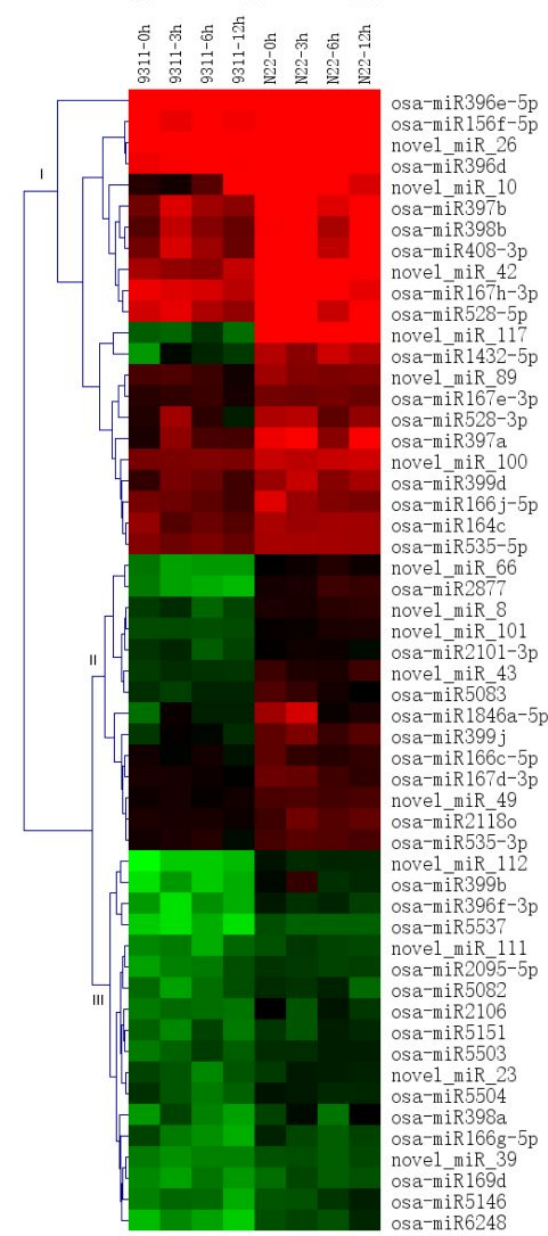

B

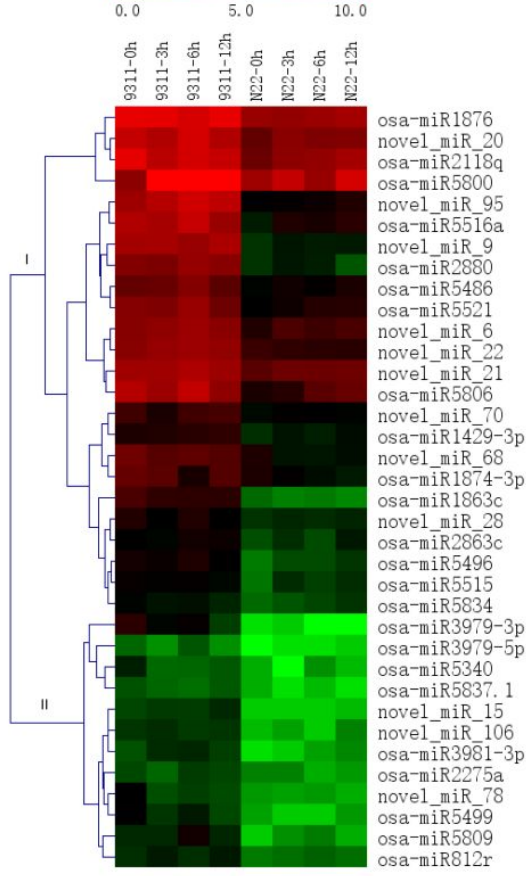

855 
$\begin{array}{lll}0.0 & 5.0 & 10.0\end{array}$

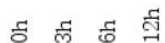
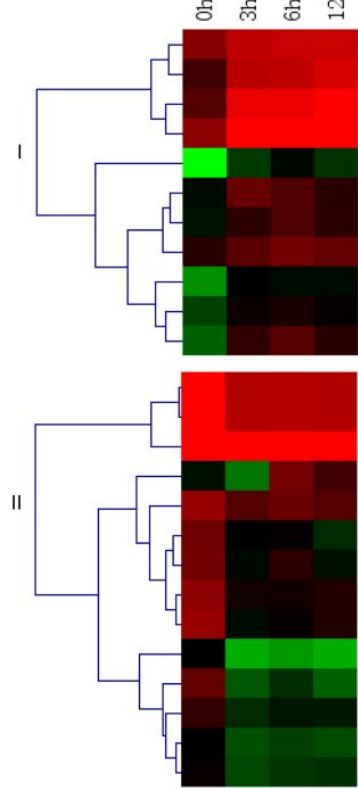

nove1_miR_35 nove1_miR_85 osa-miR579 2 osa-miR5800 nove1_miR 95 nove1_miR_14 nove1_miR_76 osa-míR515 4 nove1_miR_17 novel_miR_10.5 osa-míR6249a nove1_miR_70 nove1 miR 86 osa-miR16 $\overline{4} \mathrm{f}$ osa-miR171e-5p osa-miR164c

nove1_miR_88

osa-miR16 $\overline{6} \mathrm{k}-5 \mathrm{p}$ nove1_miR_87

osa-miR2275c osa-miR1850. 1 osa-miR171d-5p osa-miR1425-3p

novel miR 81

osa-miR2871a-5p

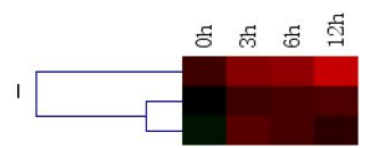

nove1_miR_22

novel miR 123 osa-míR44 $\overline{4} \mathrm{f}$

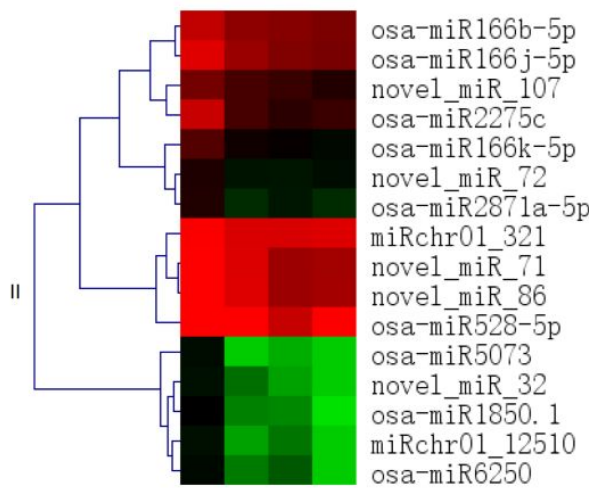

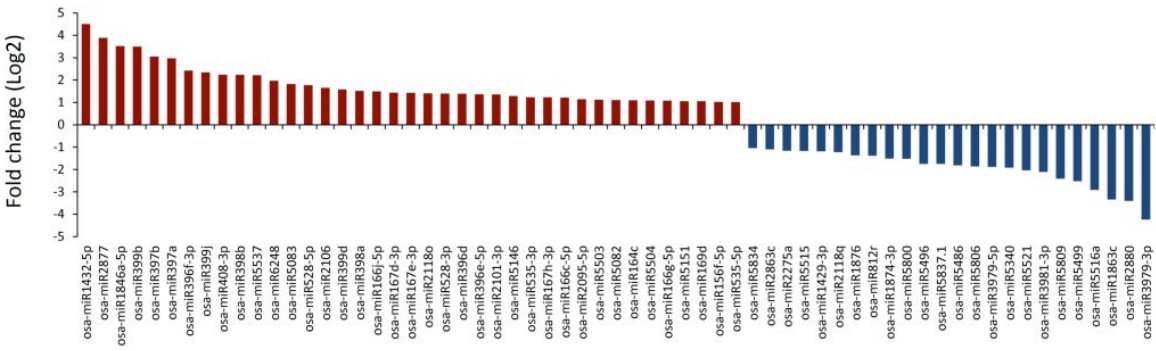

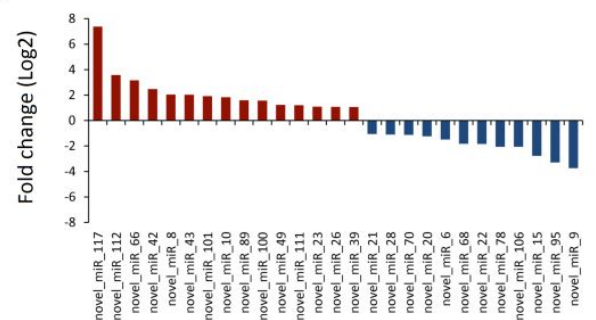


sferase activity, transferring acyl groups other transcription regulatory region DNA binding

transcription factor activity, sequence-specific

pigment binding

oxidoreductase activity, oxidizing metal ions

monovalent cation:proton antiporter activity

hydroquinone:oxygen metal ion binding

electron carrier activit

DNA binding

copper ion binding

-coumarate-CoA ligase activity
SCF ubiquitin ligase complex

SCF ubiquitin ligase complex

CCAAT-binding factor complex

apoplast

anchored component of plasma membrane

transcription, DNA-templated

SCF-dependent proteasomal ubiquitin-dependent.
response to light stimulus
regulation of transcription, DNA-templated

regulation of transcription, DNA-templated
regulation of $\mathrm{pH}$

photosynthesis, light harvesting in photosystem

photosynthesis, light harvestin

ingnin catabolic process

developmental proces

cell cycle

864

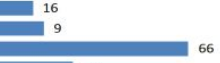

$5^{36}$

4

84

17

$17{ }^{114}$

13

175

15
15

10

8

$\int_{5}^{5}$

]$_{3}$

${ }_{8}^{5} \quad \square$ Molecular function

0

2 - $\log 10$ (PVal
B N22 Vs 9311

Plant hormone signal transduction

Phenylalanine metabolism

Photosynthesis - antenna proteins

Thiquinone and other terpenoid-quinone biosynthesis

Phenylpropanoid biosynthesis

Plant-pathogen interaction

Vitamin B6 metabolism

Insulin resistance

Protein processing in endoplasmic reticulum

Inositol phosphate metabolism

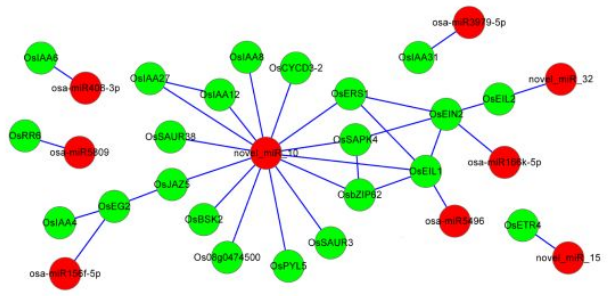




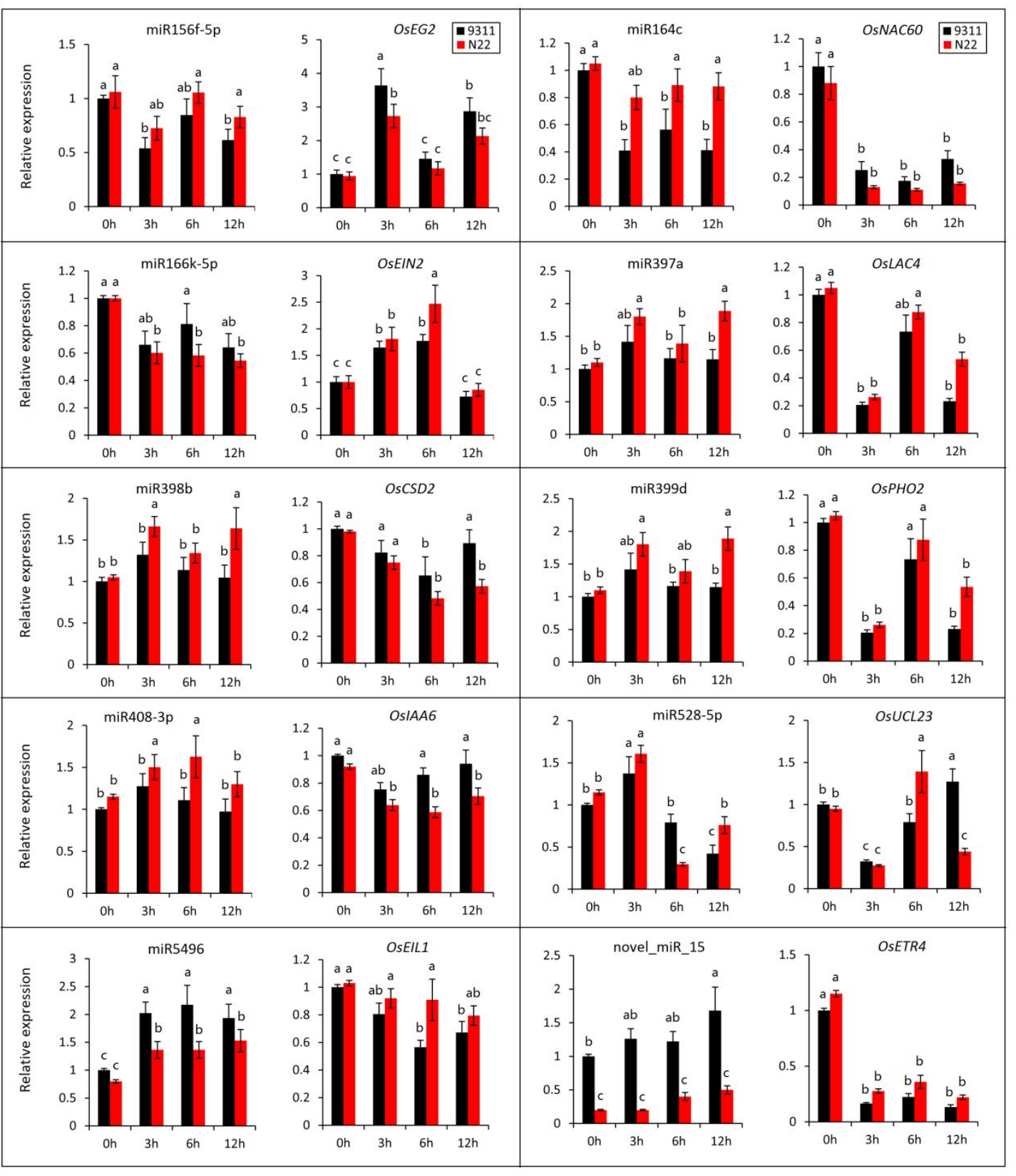

